\title{
Large pancreatic lipoma in a 69-year-old diabetic woman: diagnostic considerations
}

\author{
Agnieszka Budzyńska ${ }^{1}$, Ewa Nowakowska-Duława ${ }^{1}$, Andrzej Cholewka ${ }^{1}$, Joanna Pilch-Kowalczyk ${ }^{2}$, \\ Maciej Kajor ${ }^{3}$ \\ ${ }^{1}$ Department of Gastroenterology and Hepatology, Medical University of Silesia, Katowice, Poland \\ ${ }^{2}$ Department of Radiology, Medical University of Silesia, Katowice, Poland \\ ${ }^{3}$ Department of Pathomorphology, Medical University of Silesia, Katowice, Poland
}

Prz Gastroenterol 2014; 9 (3): 168-171

DOI: $10.5114 /$ pg.2014.43579

Key words: pancreas, lipoma, mesenchymal tumour.

Address for correspondence: Agnieszka Budzyńska MD, Department of Gastroenterology and Hepatology, Medical University of Silesia, 14 Medyków St, 40-752 Katowice, Poland, phone: +48 505036 330, +48 3278944 01, e-mail: budzynskaagnieszka@poczta.onet.pl

\begin{abstract}
Pancreatic mesenchymal neoplasms are very rare pancreatic tumours. One of them is pancreatic lipoma, often diagnosed incidentally. We herein report a case of a large lipoma of the pancreatic head, diagnosed by computed tomography and magnetic resonance imaging and confirmed by ultrasound-guided fine needle biopsy (FNA) biopsy. Regarding its benign character, silent clinical course and excellent prognosis of invasive surgical removal was avoided. We propose here the diagnostic and therapeutic management of these rare pancreatic tumours. Computed tomography is the most accurate method to diagnose pancreatic lipoma. Nevertheless large tumours may need confirmation by FNA in differential diagnosis of liposarcoma.
\end{abstract}

\section{Introduction}

Non-ductal pancreatic tumours account for 5-15\% of all pancreatic neoplasms [1]. Among them, mesenchymal tumours are recognised in only $1-2 \%$ of cases. Most mesenchymal tumours are benign, classified according to their origin as fibroma, lipoma, hamartoma, neurofibroma, schwannoma, hemangioma, myoma, he mangioendothelioma or desmoid tumour [2]. Compared to ductal adenocarcinoma, the mesenchymal tumours occur more frequently in women, are evenly distributed in the pancreas, are resectable in a higher rate and have longer survival times [3]. Herein, we report a case of a large asymptomatic pancreatic lipoma, diagnosed by contrast enhanced computed tomography (CE-CT) and magnetic resonance imaging (MRI), further confirmed by cytological examination.

\section{Case report}

A 69-year-old woman with a history of type 2 diabetes mellitus, arterial hypertension and ischaemic heart disease, and having undergone radioiodine ablation of the thyroid gland, was admitted to our department for diagnosis of a tumour localised in the head of the pancre- as. The tumour was revealed by abdominal ultrasonography performed as a routine examination. The patient had no symptoms except loosing $4 \mathrm{~kg}$ of weight in the course of a restrictive diabetic diet. Physical examination revealed obesity (body mass index (BMI) $32.2 \mathrm{~kg} / \mathrm{m}^{2}$ ). Except for high cholesterol level, the laboratory tests were in the range of reference values, including cholestatic enzymes (alkaline phosphatase, $\gamma$-glutamyl transpeptidase (GGTP) and cancer markers - particularly CA 19-9).

Abdominal ultrasonography showed the hyperechogenic normal-sized liver and stones in the gall bladder. The pancreatic mass measured $45 \mathrm{~mm}$ and was hypoechoic, with regular inside echoes and well-defined margins (Figure 1). No blood flow was recorded within the tumour on the colour Doppler test. Abdominal CE-CT showed adipose tissue density of the spherical mass involving the head and the uncinate process of the pancreas. The mass was homogenous and showed no contrast enhancement (Figure $2 \mathrm{~A}-\mathrm{C}$ ). It was surrounded by a thin rim of normally perfused pancreatic parenchyma. The main pancreatic duct was dilated to $4 \mathrm{~mm}$ proximally to the mass. On MRI the signal intensity of the pancreatic mass was equivalent to intra-ab- 
dominal and subcutaneous fat tissue (Figure $3 \mathrm{~A}-\mathrm{C}$ ). The upper part of the mass modelled the extrahepatic biliary tree without its dilation, and the lower part displaced the duodenum. Imaging methods disclosed a few moderately enlarged perihilar and periportal lymph nodes with diameters of 10-13 mm. Upper gastrointestinal endoscopy did not visualise any features compatible with outside compression or infiltration of the duodenum. Generally, the $C T$ and MR images were indicative of large lipoma of the pancreas showing weak mass effect on the neighbouring tissues. The transcutaneous ultrasound-guided fine needle aspiration biopsy (FNAB) confirmed the diagnosis, disclosing in the third sample the mature adipomatous cells with no atypia (Figure 4). The idea of surgery was abandoned due to the benign nature of the tumour and lack of clinical symptoms. The patient was advised to continue follow-up visits every 6 months.

\section{Discussion}

Lipomas are very rare pancreatic mesenchymal tumours. Until now fewer than 50 cases have been de-
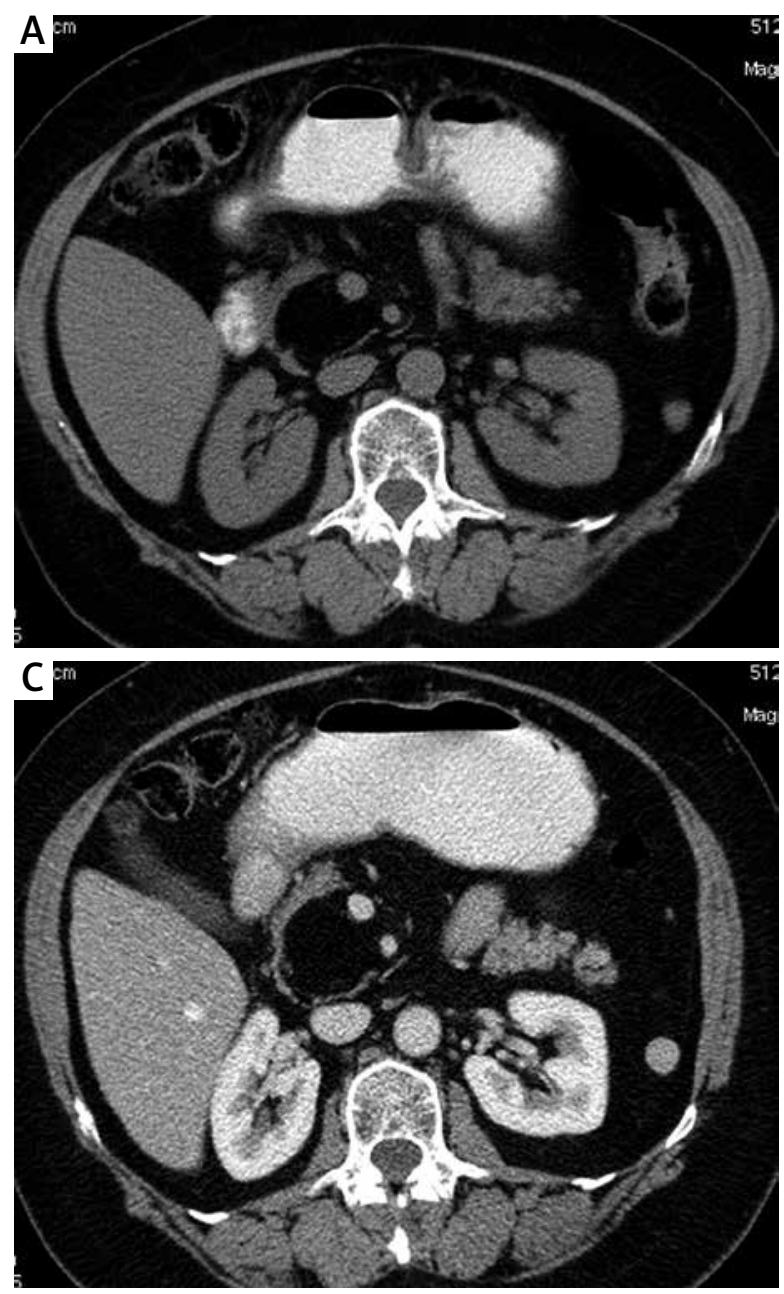

scribed, mostly as single case reports [4]. One exception is a report on 17 cases found in a retrospective analysis of CT and MRI studies performed in one radiological

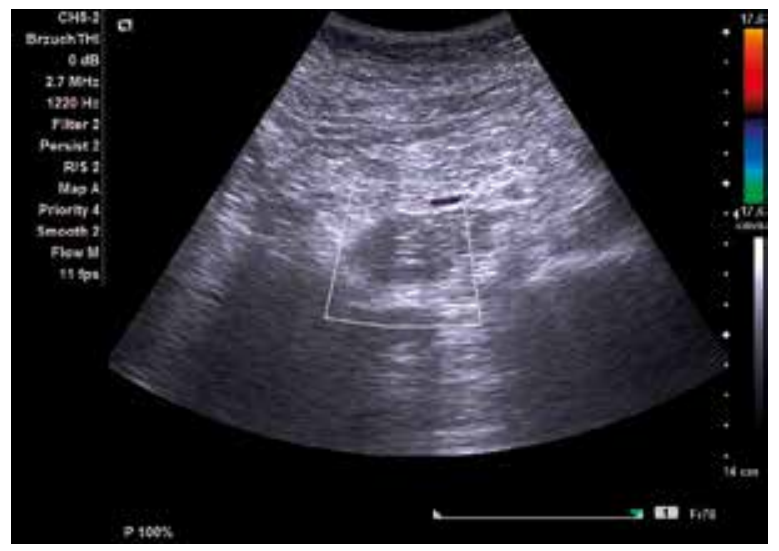

Figure 1. Abdominal ultrasonography. A homogeneous hypoechoic mass of $45 \mathrm{~mm}$ located in the head of pancreas, showing no blood flow on colour Doppler

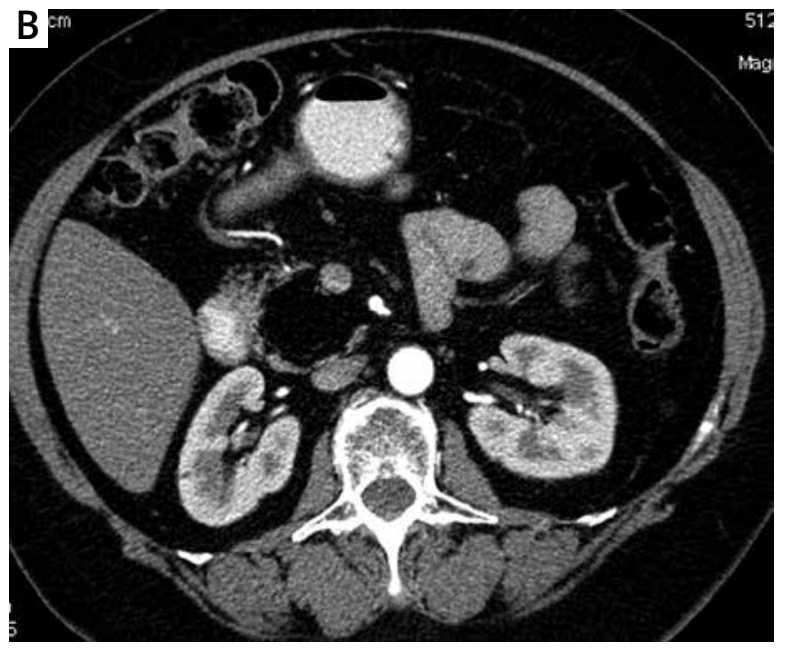

Figure 2. Contrast-enhanced CT. A hypodense homogeneous tumour, consistent with fat density (see A before contrast IV injection). The lesion is poorly vascularized (see $\mathbf{B}$ and $\mathbf{C}$ showing arterial and portal venous phases, respectively). The tumour is encapsulated and surrounded by normal pancreatic tissue. It is adjacent to the superior mesenteric vessels 

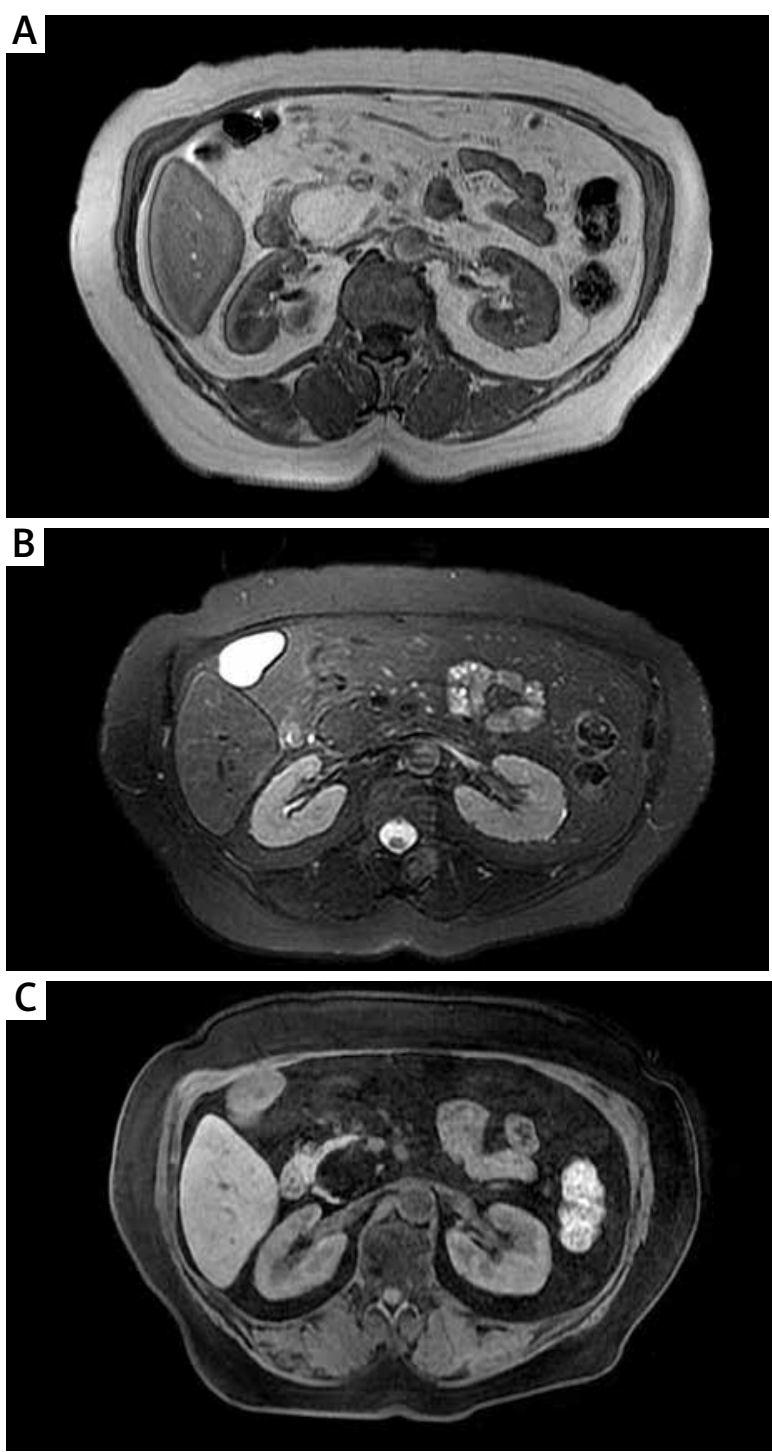

Figure 3. Magnetic resonance images. The mass of high signal intensity on $\mathrm{T} 1-(\mathbf{A})$ and T2-weighted (B) axial sequences, being isointense to subcutaneous and intra-abdominal fat. Fat-supressed T2-weighted image showing low signal intensity $(\mathbf{C})$

centre during 2 years [5]. Because of their asymptomatic clinical course, most lipomas are discovered incidentally $[4,5]$. Our patient was also referred to screening ultrasonography with no abdominal complaints. The tumour growth may be responsible for mass symptoms such as epigastric pain, jaundice, portal hypertension or acute pancreatitis [4].

Pancreatic lipomas are built of mature adipose cells, arranged in lobules and surrounded by a thin fibrous capsule separating the tumour from intact pancreatic parenchyma and peripancreatic fat. Internal septations may be seen within the tumour $[6,7]$. Lipomas are

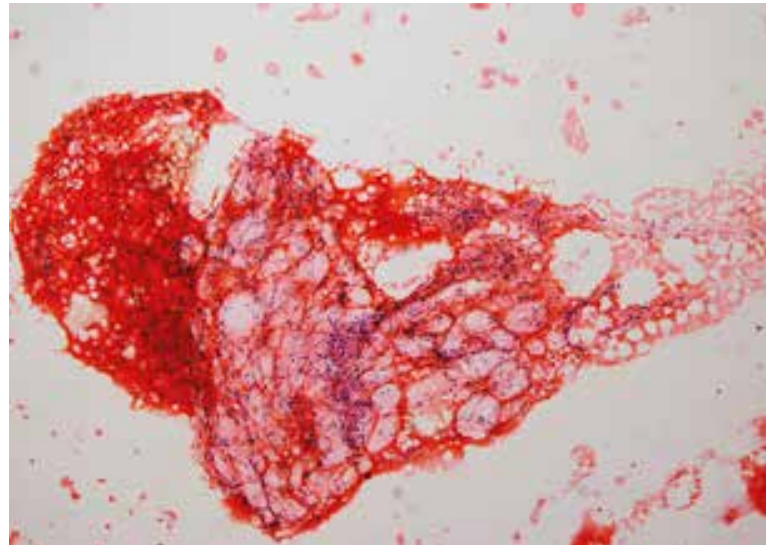

Figure 4. Histological examination reveals mature fat cells with no atypia. Staining with haematoxylin and eosin (40x)

evenly distributed in pancreatic parenchyma [4]. It is believed that lesions located in the head of the pancreas (approximately $50 \%$ of cases) arise on the early stages of embryogenesis $[5,8]$. In this setting, a portion of mesenteric or retroperitoneal fat may be trapped between the dorsal and ventral buds during the integration process. Most lipomas are less than $3 \mathrm{~cm}$ in diameter; however, giant tumours have also been reported [9].

In differential diagnosis, other lesions originating from fatty tissue should be taken into consideration, including focal fat replacement, lipomatous pseudohypertrophy and liposarcoma [10, 11]. Focal fat replacement, known also as pancreatic lipomatosis or adipose atrophy, is commonly seen in the form of fatty tissue deposition in the pancreatic parenchyma in continuity with the peripancreatic fat [5]. A fibrous capsule, typical for lipomas, is not seen. Fat gradually replenishes the atrophic pancreas, which can be seen in histological examination as a focus of pancreatic glands dispersed between the adipose tissue [12]. Lipomatous pseudohypertrophy is characterised by enlargement of the entire pancreas, caused by hypertrophy of the pancreatic fat replacing exocrine glandular tissue with preservation of the ductal system and Langerhans islets [10]. Liposarcoma is a very rare malignant tumour of the pancreas, characterised by slow growth, low metastatic potential and progressive destruction of normal pancreatic tissue. In contrast to lipoma, the liposarcoma contains poorly defined areas of higher density on CT images [11, 13]. Male sex predominance, presence of thick septations and internal calcifications within the tumour are helpful in the differentiation of liposarcoma from lipoma [13].

Based on a literature review, the diagnosis of pancreatic lipoma was mostly done via imaging techniques such as CT, MRI or endoscopic ultrasonography (EUS) $[2,4,5,14]$. The lipomas were solid and homogeneous 
tumours, showing distinct margins and no infiltration of surrounding tissues. They had a thin fibrous capsule, with tissue density ranging from -30 to $-120 \mathrm{HU}$ (Hounsfield units), and showed no contrast enhancement $[2,4,5,10,11]$. Ultrasound cannot be the reference method to diagnose pancreatic lipomas as these tumours presented either as hypo- or hyperechoic lesions [5]. Histological diagnostics were implemented in only a few cases, usually after surgical resection of the tumour. In literature, two cases were definitely diagnosed by EUS-guided FNAB [14].

The MR imaging seems to be particularly useful for the differentiation of pancreatic lipoma from focal fat replacement [15]. Lipoma has high signal intensity on both T1-weighted and T2-weighted axial sequences, and shows a homogeneous intensity pattern comparable to intra-abdominal and subcutaneous fat. Fat-supressed, T2-weighted images show homogeneous suppression of signal intensity within the tumour. The MR images, particularly with gradient recalled echo T1-weighted sequences, reveal a clear demarcation line between the mass and the adjacent peripancreatic fat.

Positron emission computerised tomography (PET$\mathrm{CT}$ ) is helpful in diagnosing pancreatic lesions, but its value in the identification of pancreatic lipomas is uncertain. Generally, the lipomas do not accumulate 2-deoxy- $\left[{ }^{18} \mathrm{~F}\right]$ fluoro-D-glucose; however, in a single case, PET provided false positive diagnosis of malignant disease, possibly due to atypical vascularisation or the presence of brown fat in the tumour $[8,16]$.

Assuming the high reliability of the imaging techniques, in most cases there is no need for histological confirmation of pancreatic lipoma, particularly if small lesions are concerned. Histopathology may be needed in patients with larger tumours, where discrimination between benign and malignant lipid containing tumours is more difficult $[6,14]$. In one reported case of giant pancreatic lipoma, the FNAB result was inconclusive and the patient underwent surgery [6]. In another case report, the first biopsy under EUS guidance provided insufficient material for examination and a second pass was needed [14]. In our case, three attempts were necessary to confirm the benign nature of the lesion. A possible explanation for the poor accuracy of FNAB is the resistance of adipose tissue to needle aspiration and the fact that the adipocytes may be easily damaged by routine use of formalin and alcohol solvents.

Among the published cases, only $12 \%$ of the lipomas were surgically resected [4]. Taking into account the benign nature and lack of clinical manifestation, most cases can escape surgical management. Surgery is only indicated in patients with symptoms resulting from the compression of the biliary tree, pancreatic duct or adjacent large vessels and in cases when the malignancy cannot be excluded. Since lipomas are encapsulated lesions, enucleation is a recommended procedure, but in some cases distal pancreatectomy or Whipple's procedure may be necessary.

In a published case report on 11 patients, the follow-up periods ranged from 3 to 72 months and no progression of the disease was found $[4,5]$. Our patient has been under surveillance for 6 months with no change in tumour size.

\section{References}

1. Warshaw A, Fernandez-del Castillo C. Pancreatic carcinoma. N Engl J Med 1992; 336: 455-65.

2. Ferrozzi F, Zuccoli G, Bova D, et al. Mesenchymal tumors of the pancreas: CT findings. J Comput Assist Tomogr 2000; 24: 622-7.

3. De Jong SA, Pickleman J, Rainsford K. Nonductal tumors of the pancreas: the importance of laparotomy. Arch Surg 1993; 128: 730-6.

4. Zhan HX, Zhang TP, Liu BN, et al. A systematic review of pancreatic lipoma: how come there are so few cases? Pancreas 2010; 39: 257-60.

5. Karaosmanoglu D, Karcaaltincaba M, Akata D, et al. Pancreatic lipoma computed tomography diagnosis of 17 patients and follow-up. Pancreas 2008; 36: 434-6.

6. Raut CP, Fernandez-del Castillo C. Giant lipoma of the pancreas: case report and review of lipomatous lesions of the pancreas. Pancreas 2003; 26: 97-9.

7. Salman Monte Z, Ruiz-Cabello Jimenez M, Pardo Moreno P, et al. Lipoma of the pancreas: diagnosis and management of these rare tumors. Rev Esp Enferm Dig 2006; 98: 884-6.

8. Hois EL, Hibbeln JF, Sclamberg JS. CT appearance of incidental pancreatic lipomas: a case series. Abdom Imaging 2006; 31: 332-8.

9. Boglina C, Inserra A, Silvano A, et al. Lipoma intrapancreatico: descrizione di un caso. Pediatr Med Chir 1993; 15: 397-9.

10. Katz DS, Hines J, Math KR, et al. Using CT to reveal fat-containing abnormalities of the pancreas. Am J Roentgenol 1999; 172: 393-6.

11. Wagliore MP, Stephens DH, Soules EH, et al. Lipomatous tumors of the abdominal cavity: CT appearance and pathologic correlation. Am J Roentgenol 1981; 137: 539-45.

12. Itai Y, Saida Y, Kurosaki Y, et al. Focal fatty masses of the pancreas. Acta Radiol 1995; 36: 178-81.

13. Kransdorf MJ, Bancroft LW, Peterson JJ, et al. Imaging of fatty tumors: distinction of lipoma and well-differentiated liposarcoma. Radiology 2002; 224: 99-104.

14. Suzuki R, Irisawa A, Hikichi T, et al. Pancreatic lipoma diagnosed using EUS-FNA. A case report. JOP 2009; 10: 200-3.

15. Secil M, Igci E, Goktay AY, et al. Lipoma of the pancreas: MRI findings. Comput Med Imaging Graph 2001; 25: 507-9.

16. Bean MJ, Fishmas EK. Focal FDG uptake in a pancreatic lipoma mimicking malignancy. J Comput Assist Tomogr 2005; 29: 475-6.

Received: 11.12 .2011

Accepted: $\quad 18.11 .2012$ 